\title{
O uso BIM para a avaliação da eficiência energética de projetos de edifícios públicos com a aplicação da
} INI-C

\author{
The BIM use for the evaluation of the energy efficiency of \\ public buildings: the building case of the Department of \\ Physics at UFPE with the application of INI-C
}

\section{Ewerton Dias Leal Pereira}

Universidade Federal de Pernambuco | Recife | Brasil | ewerton.leal@ufpe.br

Max Lira Veras Xavier de Andrade

Universidade Federal de Pernambuco | Recife| Brasil | max.andrade@ufpe.br

\section{Resumo}

Desde 2014, a Instrução Normativa SLTI/MPOG no 2, de 5 de junho de 2014, determina que obras de retrofit e projetos de edificações públicas federais obtenham a ENCE do PBE Edifica. Neste cenário, este trabalho pretende avaliar a metodologia de avaliação energética da INI-C, a partir da simulação computacional, para a determinação do nível de eficiência energética de um edifício da Universidade Federal de Pernambuco. Para esse artigo, que apresenta um trabalho ainda em desenvolvimento, espera-se avaliar como duas plataformas BIM (Revit e Archicad) são capazes de serem usadas para a avaliação da eficiência energética do edifício, a partir do INC C. Os resultados mostram que, apesar dos avanços em cada uma dessas Plataformas, estas ainda não permitem serem aplicadas integralmente na avaliação da eficiência energética de edificações públicas, atendendo à norma INI-C.

Palavras-chave: BIM. INI-C. Eficiência Energética. ENCE. Análises de Sustentabilidade.

\begin{abstract}
Since 2014, The Normative Instruction SLTI/MPOG No. 2, of June 5, 2014, determined that retrofit works and federal public building projects have to obtain the ENCE from PBE Edifica. Therefore, this article intends to apply the INI-C energy assessment methodology, based on computer simulation, to determine the level of efficiency of the original plan of the Department of Physics at UFPE, also analyzing the changes in the levels of this efficiency caused by the annexes added later, as a way of explaining the impacts of new constructions without the evaluation of the energy efficiency criterion. Then, it is proposed to evaluate the contributions that BIM can add to this process.
\end{abstract}

Keywords: BIM. INI-C. Energy Efficiency. ENCE. Sustainability Analysis.

\section{INTRODUÇÃO}

A produção de edificações que apresentem um consumo energético eficiente tem se revelado como uma estratégia congruente aos projetos de desenvolvimento de um

Como citar:

PEREIRA, E. D. L.; ANDRADE, M. L. V. X. de. O uso BIM para a avaliação da eficiência energética de projetos de edifícios públicos com a aplicação da INI-C. In: SIMPÓSIO BRASILEIRO DE TECNOLOGIA DA INFORMAÇÃO E COMUNICAÇÃO NA CONSTRUÇÃO, 3., 2021, Uberlândia. Anais [...]. Porto Alegre: ANTAC, 2021. p. 1-7. Disponível em: https://eventos.antac.org.br/index.php/sbtic/article/view/631. Acesso em: 3 ago. 2021.p.1-10. 
ambiente construído pautado na sustentabilidade e no uso eficiente das diferentes formas de energia [1]. De acordo com Lamberts, Dutra e Pereira [2] "um edifício é mais eficiente energeticamente quando proporciona as mesmas condições ambientais com menor consumo de energia". Logo, entende-se que a eficiência do consumo energético de um projeto de edifício pode impactar positivamente na construção de um ambiente urbano mais sustentável, observando que a indústria da construção civil, durante os seus processos e, enquanto edificações já construídas, configura como um dos principais consumidores de recursos energéticos. Do total de consumo de energia no Brasil, 46,5\% representa o consumo dos edifícios [3]. Além do mais, a construção de edifícios representa um consumo de $30 \%$ dos recursos naturais e $35 \%$ das emissões de carbono na atmosfera [4].

Reforça esse entendimento o fato de os custos envolvidos na operação e manutenção de um edifício ao longo de sua vida útil corresponderem a cerca de três ou quatro vezes o custo inicial de sua construção [5], e parte desse custo está relacionado ao consumo energético do edifício.

Não por acaso, diversas regulamentações estatais têm sido estabelecidas com o propósito de desenvolver e disseminar atividades que estimulem a aplicação dos conceitos relativos à eficiência energética nas edificações, reduzindo os custos operacionais nas construções e na utilização dos imóveis, como é o caso do Programa Nacional de Conservação de Energia Elétrica (PROCEL), criado pelo Ministério de Minas e Energia, em 1985 que, em 2003, tornou-se o PROCEL Edifica [6]. Em substituição ao antigo método de etiquetagem, nomeado de Requisitos Técnicos de qualidade (RTQ), em 2018, foi desenvolvida a Instrução Normativa do Inmetro para edificações comerciais (INI-C). Pela INI-C, de acordo com a Portaria no 42, do Ministério da Economia, "o novo método se baseia no consumo da energia primária de edificações a partir da utilização de dados provenientes de um metamodelo fundamentado através de redes neurais artificiais" [7], apresentando, ainda, um resultado quantitativo no qual é baseada a classificação diferente do método RTQ que resulta em um valor adimensional para definir a classificação. A INI-C teve sua última atualização pela portaria no 42, de 24 de fevereiro de 2021 do INMETRO, que passa a obrigar o uso do novo método a partir de 24 meses da publicação.

Em 2014, a Instrução Normativa no 2, lançada no Diário Oficial da União, passou a obrigar que edifícios públicos federais novos, ou em processos de reformas e ampliações, passem pelo processo de etiquetagem da PROCEL para que obtenham a Etiqueta Nacional de Conservação de Energia (ENCE).

\section{AS POTENCIALIDADES DO BIM}

Sob essa perspectiva, o BIM (Building Information Modeling) se apresenta como instrumento de auxílio à melhoria da qualidade da edificação, ao longo de todo o seu ciclo de vida, pontuando que este pode contribuir com a produção de construções mais sustentáveis [8]. As duas tecnologias que apoiam o BIM, interoperabilidade e modelagem paramétrica, são basilares para as práticas colaborativas e integradas, permitindo a constante e direta troca de informações entre os diferentes stakeholders. 
Além do mais, por meio da modelagem paramétrica é possível simular e avaliar diferentes soluções de projeto, ainda na etapa de concepção, tendo mais chance que as soluções geradas possam atender mais facilmente aos critérios de sustentabilidade estabelecidos [9], possibilitando processos mais inteligentes de correção de sistemas em funcionamento, bem como, permitindo a reunião de informações capazes de auxiliar o processo decisório de projeto, tornando mais fácil a obtenção de selos de certificação de sustentabilidade e de eficiência energética [10]. Por meio de uma prática de projeto integrada, os profissionais de diferentes áreas poderão participar mais efetivamente do processo projetivo, gerando soluções que contribuam para a definição de escolhas mais assertivas e eficientes, situação que também pode beneficiar a eficiência energética da edificação.

Estudando a adoção do BIM para a sustentabilidade, Chong et al. [10] identificaram e analisaram uma gama de trabalhos para diferentes categorias do ciclo de vida da edificação. Neste trabalho, os autores supracitados identificaram que o BIM pode ser aplicado em todas as três definições de sustentabilidade: a ambiental, social e econômica. A pesquisa constatou que a fase de projeto é a fase na qual são mais exploradas as soluções para atendimento às definições de sustentabilidade, na medida em que envolve a concepção e desenvolvimento do projeto do objeto a ser construído, com uma maior quantidade de diferentes atores.

Chong et al. [10] também destacam que a utilização do BIM pode ser considerada uma das formas mais efetivas de se reduzir as emissões de carbono no ambiente construído, haja vista que os benefícios que o BIM adiciona às diferentes categorias citadas possibilita a renovação de construções existentes apoiada em análises sistemáticas que promovem a escolha das soluções mais assertivas, considerando que o processo de renovação de edificações se configura como aquele que necessita de uma acurácia específica, observando que o impacto em algo previamente construído, por vezes secular, pode ser irrecuperável.

Já Freire e Amorim [11] estudaram a afinidade dos não especializados com a tecnologia e os softwares BIM. Esses autores afirmam que muitos dos conhecimentos em BIM são insuficientes para simular as soluções que levem em consideração a eficiência energética da edificação. Tem-se que os programas mais conhecidos são empregados apenas nas etapas finais do processo de projeto, momento no qual as alterações no projeto são mais custosas e representam pouco impacto nas soluções iniciais. Por outro lado, ferramentas usadas nas simulações da eficiência energética das edificações são cada vez mais comuns na etapa de concepção e geração do partido, alguns dos quais usam plugins para programas, como o Rhino para o Grasshopper. Esses têm possibilitado simulações na etapa de concepção, permitindo realizar múltiplas avaliações de desempenho das soluções inicialmente concebidas.

É neste contexto que o presente artigo se insere. Este faz parte de uma pesquisa de iniciação científica, ainda em desenvolvimento, que propõe o desenvolvimento e a experimentação de um método de projeto que empregue o BIM na geração de relatórios de avaliação da classificação de eficiência energética de edificações públicas a partir da metodologia proposta pela Instrução Normativa do Inmetro C (INI-C). Para 
experimentar o método, esta pesquisa usa para testes o edifício do Departamento de Física da Universidade Federal de Pernambuco (UFPE).

A primeira etapa do trabalho abarcou a realização de uma investigação do panorama atual do uso BIM para a sustentabilidade, com foco nas análises de eficiência energética. Além disso, foi realizada uma investigação sobre as principais modificações entre os métodos de etiquetagem brasileiros do PBE Edifica, o RTQ e o INI para compreender e aplicar a metodologia mais recente de classificação da eficiência energética.

\section{A PROPOSTA}

O método a ser desenvolvido visa auxiliar a decisão do projeto de arquitetura, a fim de contribuir para a geração de soluções mais eficientes energeticamente. Parte-se para a criação explícita e aplicável de um método para a resolução de um problema. Para a condução desta pesquisa, está se usando como método o Design Science Research (DSR). Esse é usado em pesquisas que visam desenvolver e projetar soluções que auxiliem na melhoria de sistemas existentes, na resolução de problemas e na criação de novos artefatos de auxílio à melhoria da atuação humana [12], utilizando-se das etapas de conscientização, sugestão e desenvolvimento de um artefato.

A primeira etapa do DSR consistiu na conscientização do problema, nesta fase foi realizada uma Revisão Sistemática da Literatura (RSL), a fim de aprofundar os conhecimentos na área. A etapa seguinte foi a escolha de um edifício para experimentar o método. As próximas etapas, ainda em desenvolvimento, são: Modelagem BIM do edifício do Departamento de Física da UFPE, pautando-se nos projetos básicos de arquitetura; Análises da eficiência energética do edifício original e das várias ampliações; Discussão de como o BIM poderia auxiliar na avaliação da eficiência energética da edificação, de modo rápido e eficiente, contribuindo, inclusive, na melhor classificação da eficiência energética da edificação, baseada no método INIC; Mostrar como as simulações em BIM podem auxiliar nas análises das eficiências energéticas das ampliações do edifício em estudo, como possíveis impactos nas decisões de projeto arquitetônico, de modo a gerar projetos mais eficazes e sustentáveis.

A pesquisa tem foco na utilização dos métodos de combinação BIM-BEM denominados de integrated. $O$ método integrated é baseado no processo dos dados dentro do próprio software, enquanto o run-time interoperable é formulado a partir da utilização de plugins ou APIs [13]. A escolha de um método pelo qual não seja necessária a exportação entre diferentes softwares visa facilitar a avaliação, a fim de estudar a possibilidade de maior adesão ao processo, haja vista a dificuldade de implementação em Plataformas BIM, em singular.

Para o estudo apresentado neste artigo serão analisados os softwares Revit 2021 e Archicad 23, com seus simuladores de eficiência energética padrão, o DOE 2.2 e o EcoDesigner Star, respectivamente. 


\section{APLICAÇÃO DA INI-C E INTEGRAÇÃO COM O BIM}

Identificados os blocos tratados como anexos à edificação original, está sendo empregado o método da simulação previsto na INI-C, entendendo que a forma da edificação, bem como a sua organização interna, podem interferir na acurácia possibilitada pelo método simplificado do INI-C. Além disso, tem-se que o método da simulação pode potencializar a utilização do BIM ao propor que o modelo seja utilizado para além do fornecimento de informações básicas como áreas e volumes e passe a utilizar os metadados agregados para a geração de informações complexas ao final do processo. Pontua-se, também, que um modelo BIM adequado pode ser utilizado para simulações não possíveis de serem estimadas pelo método simplificado, como a questão da ventilação natural, situação que não será testada nesta pesquisa.

É importante destacar que o edifício estudado apresenta diversos laboratórios com equipamentos específicos. No entanto, para o estudo do edifício, está sendo feita uma análise simplificada de modo a atender o escopo do trabalho. Deve-se pontuar que as diferentes formas de ocupação do espaço pelos seus usuários têm papel relevante na questão do desempenho térmico da edificação, como explica Coelho [14], situação que não está sendo considerada nesta pesquisa.

O método de simulação da INI-C necessita da modelagem de uma edificação de referência, que apresente a mesma geometria da edificação real (excluindo elementos de proteção solar como brises), mas que tenha parâmetros pré-estabelecidos na norma, a fim de determinar a sua classificação como " $D$ ", e, desta forma, classificar o quão mais eficiente ou menos eficiente a edificação com seus parâmetros reais se apresenta frente à edificação de referência, tendo a edificação real modelada todos os seus elementos, incluindo os de proteção solar. A partir disso será feita a classificação da edificação considerando seu projeto original e as diferentes ampliações realizadas. Então, serão comparados os dados obtidos em cada fase do edifício. Por fim, serão simuladas alterações pontuais em cada uma das fases, visando identificar possíveis melhorias na eficiência energética da edificação.

\section{RESULTADOS}

A INI-C exige uma lista de atributos que os softwares de avaliação necessitam abranger para que seja apto a realizar as simulações da norma, sendo uma das mais importantes a validação pela ASHRAE Standard 140, a qual o simulador do Archicad, explicitamente, indica possuir.

A atribuição dos dados necessários para a simulação energética padrão em ambos os softwares, Revit e Archicad, não apresenta dificuldades significativas quando utilizados os materiais e elementos padrões, já pré-configurados, os quais apresentam propriedades já definidas, situação que permite agilidade e facilidade na execução da simulação. É necessário apenas configurar as condições de uso, ocupação e o sistema de ventilação de cada ambiente, situação que no Archicad é feita a partir da própria ferramenta "zona", enquanto no Revit é necessário a utilização da função "espaço", 
não tão recorrente na modelagem comum da edificação, quando se utiliza a função "ambiente".

No entanto, a atribuição das propriedades que são necessárias para avaliação da edificação de referência solicitada pela INI-C tornam o processo complexo e confuso. Isso porque ambos os programas calculam automaticamente valores como a transmitância térmica (U) a partir dos dados padrões agrados aos materiais préconfigurados, baseando-se também na espessura modelada em projeto para as diferentes camadas de um elemento construtivo como uma parede ou um teto, situação que exige que sejam alterados os dados reais dos materiais até que os cálculos automáticos atinjam os valores exigidos pela norma para a transmitância, já que esta é calculada a partir espessura da camada do material (indicada pela norma) e da sua condutividade térmica. Nesse sentido, o Revit permite sobrepor completamente esses valores apenas para avaliação térmica, o que evita a remodelagem da edificação com os parâmetros de referência da INI-C, situação necessária no Archicad. Entretanto, a configuração de sobreposição de propriedades térmicas padrão para elementos construtivos do Revit não atinge, nem se aproxima, da exigida pela norma, questão que exige a alteração contínua das propriedades dos materiais até que se atinja o valor exigido pela norma.

Figura 01 : Janela de sobreposição de propriedades térmicas dos elementos construtivos no REVIT

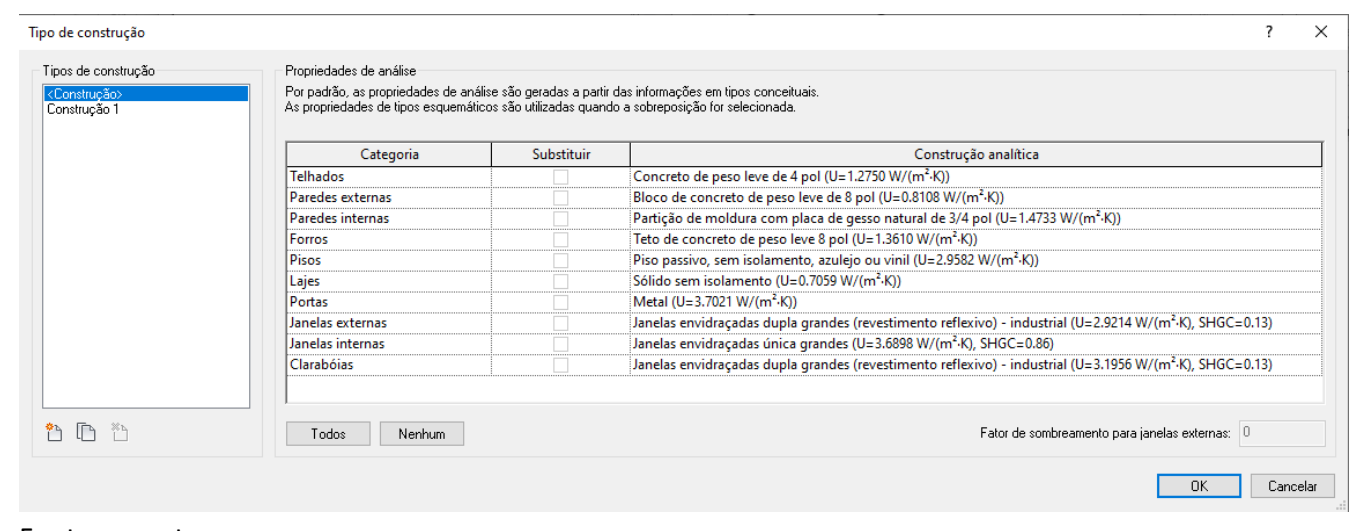

Fonte: os autores.

Outra propriedade que não é possível alterar como a norma solicita é a absortância, sendo utilizada a absorvência solar em ambos os softwares. A norma também exige um percentual máximo de aberturas na fachada de $40 \%$, situação não controlável no Archicad a não ser que seja adequado manualmente cada uma das esquadrias. Sobre esse item, o REVIT permite sobrepor esse valor quando é exportado para a extensão Gbxml, mas não permite enquanto simulação no próprio software. Ainda sobre as esquadrias, o REVIT não permite alterar facilmente as propriedades de material das mesmas, nem as selecionar no modelo energético simplificado, enquanto no Archicad a visualização das propriedades de todos os materiais é explícita em uma única janela. 
Figura 02: Visualização das propriedades dos materiais no Archicad

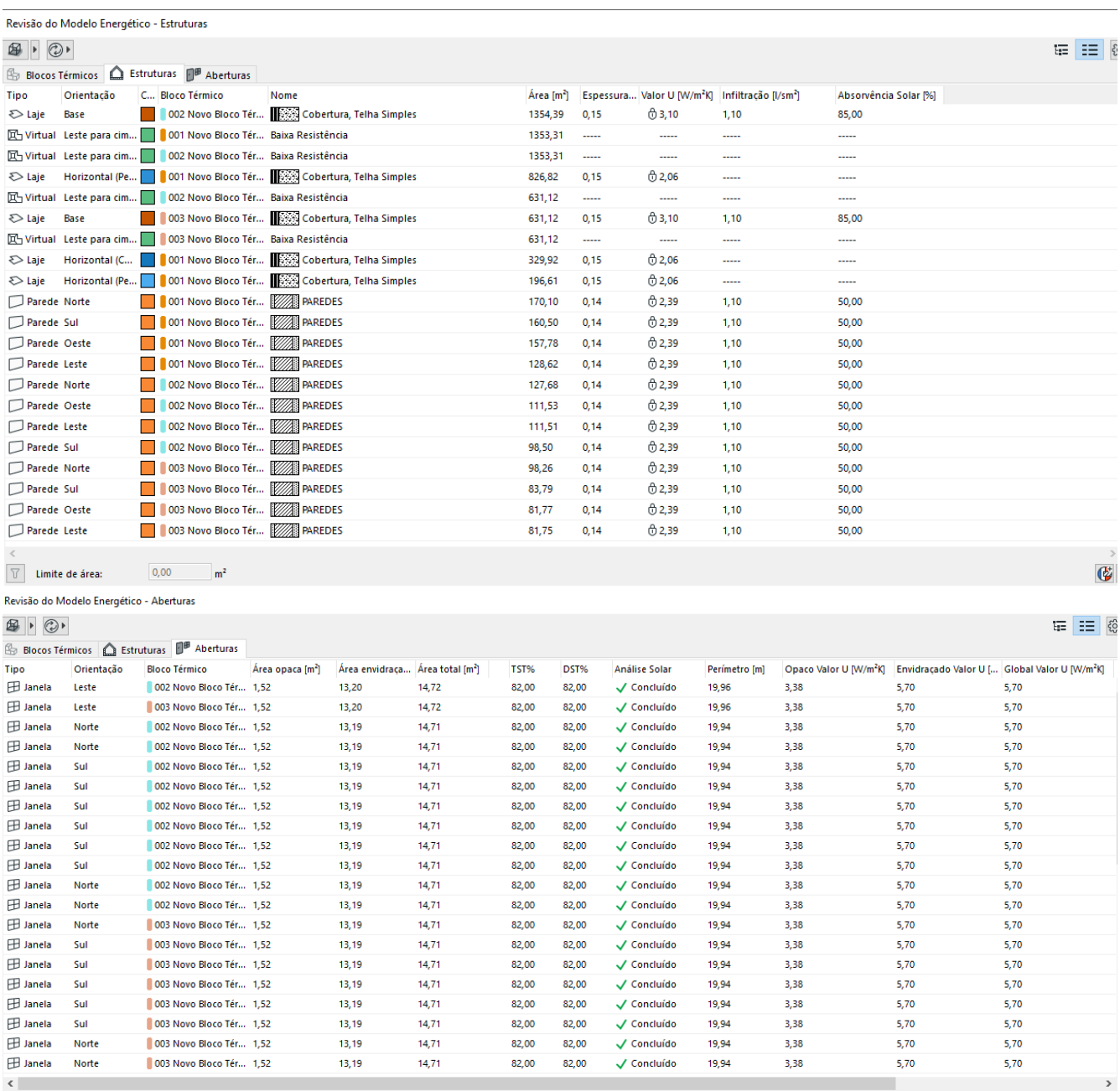

Fonte: os autores.

Figura 03 : Janela de exportação para gbXML do Revit permite sobrepor a porcentagem de área de aberturas

\begin{tabular}{|c|c|c|c|}
\hline Configurações avançadas de energia & & & \\
\hline Parâmetro & Valor & & $\wedge$ \\
\hline Modelo detalhado & & $\hat{\imath}$ & \\
\hline Percentual de destino da vidraça & $0 \%$ & & \\
\hline Altura de destino do parapeito & 0.7500 & & \\
\hline A vidraça está sombreada & $\square \times$ & & \\
\hline Profundidade da sombra & 0.4572 & & \\
\hline Percentual de claraboias do destino & $0 \%$ & & \\
\hline Largura e profundidade da \&clarabóía & 0.9144 & & \\
\hline Dados de construção & & $\hat{\imath}$ & \\
\hline Tipo de construção & Escola ou Universidade & & \\
\hline Tabela de operaçốes de construção & Padrão & & \\
\hline Sistema AVAC & Ventilação/Ar-condicionado central, Ac & riador 5,9 & \\
\hline Informaçôes sobre o ar exterior & Editar.... & & \\
\hline Ambiente/Espaço de dados & & $\hat{\imath}$ & \\
\hline Exportar categoria & Ambientes & & \\
\hline Propriedades térmicas do material & & $\hat{\imath}$ & \\
\hline Tipos conceituais & Editar... & & \\
\hline Tipos esquemáticos & <Construção> & & \\
\hline Flementos detalhados & $\Pi$ & & 2 \\
\hline \multicolumn{4}{|l|}{$\begin{array}{l}\text { Como estas confiquracôes afetam a análise de } \\
\text { energia? }\end{array}$} \\
\hline & & Cancelar & \\
\hline
\end{tabular}

Fonte: os autores. 
Ambos os softwares permitem a configuração de uso e ocupação adequados à norma, enquanto o REVIT ainda permite atribuir porcentagens de um valor total a cada hora do dia, indicando, por exemplo, que de 1 pessoa por $\mathrm{m}^{2}$ quadrado total, no horário de $12 \mathrm{~h}$ estarão $100 \%$ das pessoas possíveis, enquanto às $8 \mathrm{~h}$ estarão apenas $30 \%$ dessas, situação que a norma não exige, mas não é possível no Archicad.

Por sua vez, o Archicad permite a alteração dos parâmetros de condicionamento de ar tal qual a norma exige como referência para o Coeficiente de Performance (COP) e a temperatura setpoint, enquanto não foi possível alterar o COP no Revit.

\section{DISCUSSÕES}

Os resultados observados até o momento confirmam a continuidade de dificuldades já observadas pela bibliografia analisada, sobretudo em relação às questões referentes aos diferentes padrões de simulação que são desenvolvidos para cada uma das diferentes Plataformas BIM testadas neste trabalho. Compreende-se que as diferentes normativas de avaliação, como a LEED e o selo AQUA carregam suas particularidades [15], bem como a INI-C também apresenta outra proposta para o processo de simulação. É indubitável que as Plataformas BIM, tratando-se de ferramentas de abrangência global, podem não compreender todos os parâmetros necessários a todos os processos de simulação, fazendo-se necessárias adaptações no processo, ou adições de ferramentas que auxiliem o BIM a ser útil ao padrão de etiquetagem brasileiro, como experimentam Bracht, Melo e Lamberts [13] ao estudar a utilização da exportação de arquivos gbXML do Revit, Openbuildings e do Archicad aplicando as informações recolhidas do gbXML advindo do modelo BIM a um metamodelo que os próprios autores desenvolveram.

Igualmente, a divulgação da norma INI-C é extremamente recente, tendo sua obrigatoriedade de aplicação ainda futura, situação que irá evidenciar a necessidade de alterações a partir da difusão do seu uso e aplicação. Esta é uma questão que ratifica a necessidade da pesquisa nessa área, haja vista ser prudente antecipar as avaliações de eficiência energéticas, ainda nas etapas iniciais do projeto, de modo a refinar o processo de concepção, e, possivelmente, tornar a análise mais acessível ao projetista comum.

Vale pontuar que, diferente do método simplificado, pelo qual o usuário tem total controle de onde estão sendo empregado cada um dos valores recolhidos, os cálculos do método de simulação são realizados pelo software, o que encobre o processo de aplicação dos dados inseridos, situação que não permite validar facilmente se os cálculos estão sendo realizados em consonância com o que a norma instrui, fato esse que pode apresentar discrepância entre os diferentes métodos e diferentes softwares durante o processo de classificação da edificação.

Por fim, reconhece-se que, mesmo que determinadas informações precisem ser recorrentemente inseridas nas Plataformas BIM, como questões relativas às 
regionalidades bioclimáticas, os metadados atrelados ao modelo BIM se apresentam aptos a proporcionar uma análise de eficiência energética adequada à etiquetagem de edificações, considerando que, a depender das particularidades de cada método, serão exigidas diferentes informações agregadas ao modelo.

Cabe, nas próximas etapas dessa pesquisa, experimentar os métodos de simulação no projeto do edifício do departamento de Física da UFPE. Por meio da experimentação nesse edifício espera-se, comprovar ou não, a eficácia das Plataformas Revit e Archicad para a avaliação da eficiência energética desse edifício e suas ampliações segundo a norma INI-C pelo método de simulação computacional.

\section{REFERÊNCIAS}

[1] LAI, Chun Sing; JIA, Youwei; DONG, Zhekang; WANG, Dongxiao; TAO, Yingshan; LAI, Qi Hong; WONG, Richard T. K.; WU, Ahmed F. Zobaa; Ruiheng; LAI, Loi Lei. A Review of Technical Standards for Smart Cities. MDPI: Clean Technologies, Basiléia, Suíça, v. 2, n. 3, p. 290-310, jun. 2020. DOI: https://doi.org/10.3390/cleantechnol2030019. Disponível em: https://www.mdpi.com/2571-8797/2/3/19. Acesso em: 20 set. 2020.

[2] LAMBERTS, R.; DUTRA, L.; PEREIRA, F. Eficiência Energética na Arquitetura. 3. ed. São Paulo: PW, 1997. 192 p.

[3] EMPRESA DE PESQUISA ENERGÉTICA (Brasil). Balanço Energético Nacional: 2019. Relatório Síntese / Ano Base 2018. Rio de Janeiro, 2019. Disponível em: https://www.epe.gov.br/pt/publicacoes-dados-abertos/publicacoes/balanco-energeticonacional-2019. Acesso em: 16 nov. 2020.

[4] LEITE, Ellen Flávia Weis; HACKENBERG, Ana Mirthes. Eficiência energética de envoltória: comparação dos métodos de avaliação RTQ-C e INI-C através de estudo de caso. Revista gestão e sustentabilidade ambiental, Florianópolis, v. 9, p. 532-552, fev. 2020. DOI: http://dx.doi.org/10.19177/rgsa.v9e02020532-552.

[5] DAVIS, Daniel. Modelled on Software Engineering: Flexible Parametric Models in the Practice of Architecture. 2013. 243 f. Tese (Doutorado em Filosofia) - School of Architecture and Design College of Design and Social context, RMIT University, Melbourne, Austrália, set. 2013. Disponível em: https://www.danieldavis.com/papers/danieldavis_thesis.pdf. Acesso em: 16 jun. 2020.,

[6] BRASIL. MINISTÉRIO DO DESENVOLVIMENTO, INDÚSTRIA E COMÉRCIO EXTERIOR. Instituto Nacional de Metrologia, Qualidade e Tecnologia. Portaria no 50, de 01 de fevereiro de 2013. Brasília, DF: Ministério do Desenvolvimento, Indústria e Comércio Exterior, 01 fev. 2013. Disponível em: https://www.pbeedifica.com.br/sites/default/files/projetos/etiquetagem/residencial/do wnloads/RAC.pdf. Acesso em: 12 nov. 2020.

[7] BRASIL. MINISTÉRIO DA ECONOMIA. INSTITUTO NACIONAL DE METROLOGIA, Qualidade e Tecnologia. Portaria № 42, de 24 de fevereiro de 2021. Brasília, DF, 24 fev. 2021. Disponível em: http://www.inmetro.gov.br/legislacao/rtac/pdf/RTAC002707.pdf. Acesso em: 05 mar. 2021.

[8] EASTMAN, Chuck; TEICHOLZ, Paul; SACKS, Rafael; LISTON, Kathleen. BIM handbook: A guide to building information modeling for owners, managers, designers, engineers and contractors. 2. ed. New Jersey: John Wiley \& Sons, 2011. ISBN: 978-0-470-18528-5.

[9] NAKAMURA, K. Natália; CARLO, C. Joyce. Introdução ao uso do sistema BIM (building information modeling) para simulação termo-energética. Cadernos PROARQ, Rio de Janeiro, n. 21, p. 107-119, dez. 2013. Disponível em: 
https://cadernos.proarq.fau.ufrj.br/public/docs/cadernosproarq21_pt.pdf. Acesso em: 20 de set. 2020.

[10] CHONG, Heap-Yih; LEE, Cen Ying; WANG, Xiangyu. A mixed review of the adoption of Building Information Modeling for sustainability. Journal of Cleaner Production, v. 142, p. 4114-4126, jan. 2017. DOI: https://doi.org/10.1016/j.jclepro.2016.09.222.

[11] FREIRE, Márcia; AMORIM, Arivaldo. A abordagem BIM como contribuição para a eficiência energética no ambiente construído. In: ENCONTRO DE TECNOLOGIA DA INFORMAÇÃO E COMUNICAÇÃO, 5, 2011, Salvador. Anais [...]. Salvador: Universidade Federal da Bahia, 2011. p. 1-12.

[12] LACERDA, Daniel P.; DRESCH, Aline; PROENÇA, Adriano; ANTUNES JÚNIOR, José A. V.. Design Science Research: método de pesquisa para a engenharia de produção. Gestão \& Produção, São Carlos, v. 20, n. 4, p. 741-761, nov. 2013. DOI:

https://doi.org/10.1590/S0104-530X2013005000014. Disponível em: https://www.scielo.br/j/gp/a/3CZmL4JJxLmxCv6b3pnQ8pq/?lang=pt. Acesso em: 10 jun. 2020.

[13] BRACHT, M.K., MELO, A.P.; LAMBERTS, R.. A metamodel for building information modeling-building energy modeling integration in early design stage. Automation in Construction, vol. 121, n. 103445, p. 01-19, jan. 2021. DOI: https://doi.org/10.1016/j.autcon.2020.103422.

[14] COELHO, Alice de Castro Cantele. A utilização do BIM na análise da Eficiência Energética de Edifícios: Ferramentas e Interoperabilidade. 2020. 82 f. Dissertação (Mestrado em Construção e Reabilitação Sustentáveis) - Escola de Engenharia, Universidade do Minho, Braga, Portugal, jan. 2020. Disponível em: http://repositorium.sdum.uminho.pt/handle/1822/67090. Acesso em: 10 jun. 2020.

[15] ZEMERO, Bruno Ramos. Análise da aplicabilidade da tecnologia BIM em projetos sustentáveis e etiquetagem de edificações no Brasil. 2016. 183 f. Dissertação (Mestrado do Programa de Pós-graduação em Arquitetura e Urbanismo) - Universidade Federal do Pará, Belém, Brasil, 2016. Disponível em: https://ppgau.propesp.ufpa.br/ARQUIVOS/documentos/Dissertacao\%20\%20Bruno\%20Zemero.pdf. Acesso em: 05 fev. 2021. 